\title{
Accessing Excited State Molecular Vibrations by Femtosecond Stimulated Raman Spectroscopy
}

\author{
Giovanni Batignani, Carino Ferrante, and Tullio Scopigno*
}

Cite This: J. Phys. Chem. Lett. 2020, 11, 7805-7813

Read Online

ABSTRACT: Excited state vibrations are crucial for determining the photophysical and photochemical properties of molecular compounds. Stimulated Raman scattering can coherently stimulate and probe molecular vibrations with optical pulses, but it is generally restricted to ground state properties. Working under resonance conditions enables cross-section enhancement and selective excitation to a targeted electronic level but is hampered by an increased signal complexity due to the presence of overlapping spectral contributions. Here, we show how detailed information about ground and excited state vibrations can be disentangled by exploiting the relative time delay between Raman and probe pulses to control the excited state population, combined with a diagrammatic formalism to dissect the pathways concurring with the signal generation. The proposed method is then exploited to elucidate the vibrational properties of the

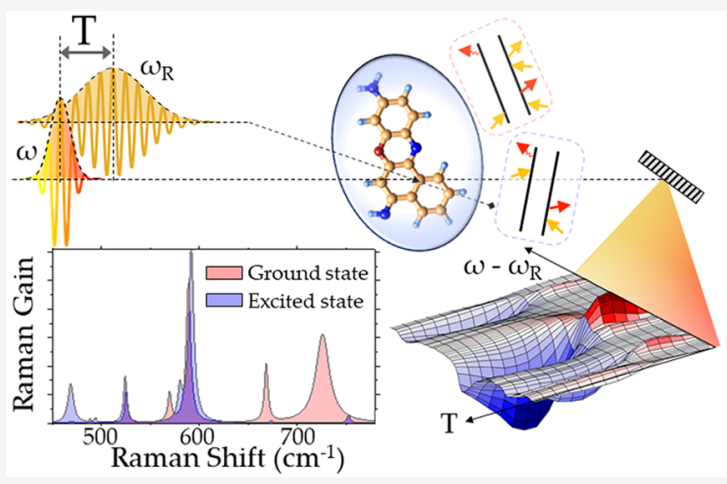
ground and excited electronic states in the paradigmatic case of cresyl violet. We anticipate that the presented approach holds the potential for selective mapping of the reaction coordinates pertaining to transient electronic stages implied in photoactive compounds.

$\mathrm{R}$ aman spectroscopy is a powerful tool for accessing the vibrational fingerprints of molecules or solid state compounds and can be used to extract structural and dynamical information about the samples being investigated. During the past several decades, because of the development of ultrafast and nonlinear optical techniques, ${ }^{1}$ different experimental protocols based on coherent Raman scattering ${ }^{2}$ have been introduced for investigating the vibrational properties of reacting species and for studying their photophysical and photochemical properties. Particular efforts have been devoted to the development of experimental and theoretical protocols that can measure and assign vibrational modes on excited potential energy surfaces, distinguishing them from ground state eigenstates. ${ }^{3-12}$

Femtosecond stimulated Raman scattering (FSRS) represents a convenient way to combine the vibrational sensitivity of Raman spectroscopy with the efficiency of coherent processbased techniques, providing high-intensity and fluorescence background free signals. ${ }^{13}$ FSRS exploits the combination of a narrowband Raman pulse (RP) with a femtosecond probe pulse (PP) to coherently stimulate and probe vibrational excitations, read out as Raman gain on the high directional PP field and probed over a wide spectral range. Hence, the stimulated Raman spectrum can be isolated considering the ratio $\mathrm{RG}=\frac{I_{\mathrm{O}}(\omega)}{I_{\mathrm{Off}}(\omega)}$ between the PP spectrum measured in the presence $\left(I_{\mathrm{On}}\right)$ and absence $\left(I_{\text {Off }}\right)$ of the RP, conventionally termed the Raman gain, providing high structural sensitivity ${ }^{14-16}$ to the sample under investigation. The use of a broadband probe pulse provides the

chance to access the entire vibrational spectrum, from low- to high-frequency Raman modes, in a single acquisition. Additionally, FSRS is immune to the non-vibrationally resonant background, which on the contrary can overwhelm the vibrational response measured by different frequency-domain nonlinear Raman experimental layouts, such as coherent antiStokes Raman scattering, in both off-resonant and resonant regimes. ${ }^{2,17,18}$ Moreover, because FSRS can combine a high spectral resolution with a femtosecond time precision in the stimulation of Raman coherences, ${ }^{19,20}$ adding a third pulse, namely the photochemical pump that precedes the RP-PP pair and excites the sample, turns FSRS into a pump-probe technique $^{21-23}$ that can access vibrational spectroscopy on subpicosecond time regimes, ensuring at the same time atomic spectral resolution. ${ }^{24-37}$

Notably, Raman features in the FSRS scheme provide information about the imaginary part of the generated nonlinear polarization, due to the self-heterodyne nature of the measured signal. ${ }^{2,13,38}$ Hence, the spectral profiles can result in complex line shapes, depending on the probed spectral range, on the $\mathrm{RP}$

Received: June 26, 2020

Accepted: August 25, 2020

Published: August 25, 2020 

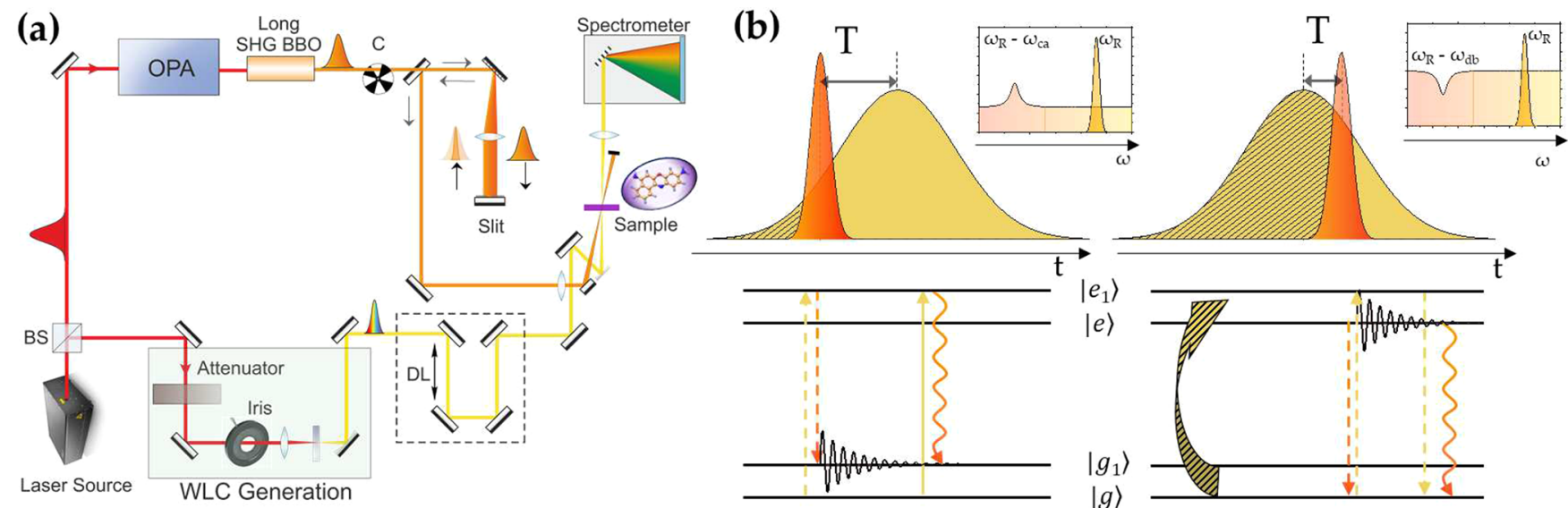

Figure 1. Two-pulse FSRS concept in the presence of a resonant Raman pulse. (a) Sketch of the experimental setup and (b) pulse interaction scheme. For a PP preceding the RP, maximum vibrational coherences are stimulated in the ground electronic state and result in positive gains on the PP spectrum. On the contrary, for a RP preceding the PP, a large portion of the Raman pulse can be absorbed by the system, and excited state properties can be probed by subsequent FSRS interactions with the RP-PP pair. In the bottom of panel b, we report energy ladder diagrams describing the two different processes. $|g\rangle$ and $\left|g_{1}\right\rangle$ denote the ground and the first vibrational excited levels in the electronic ground state, respectively, while $|e\rangle$ and $\left|e_{1}\right\rangle$ indicate their counterparts in the excited electronic level. Abbreviations: BBO, beta barium borate; BS, beam splitter; DL, delay line; OPA, optical parametric amplifier; SHG, second harmonic generation.

resonance condition, and on the order of the radiation-matter interactions that generate the nonlinear Raman signal. ${ }^{13,39}$ Specifically, for a RP wavelength $\lambda_{\mathrm{RP}}$ tuned far from any electronic transition of the system, FSRS bands appearing to the red side of the spectrum (at PP wavelengths $\lambda$ larger than $\lambda_{\mathrm{RP}}$ ) are always positive gains, while FSRS features to the blue side are negative losses. ${ }^{40}$ On the contrary, for a resonantly tuned RP, the FSRS signals appearing to the blue side of the spectrum show a profile evolving from negative losses to positive gains through dispersive line shapes as a function of the resonance condition, ${ }^{39}$ while FSRS bands to the red side typically show positive profiles. Critically, in the presence of a resonant RP, Raman coherences can be generated on different potential energy surfaces; therefore, assigning the measured vibrations to the pertinent electronic state can represent a demanding task. In particular, while the resonance enhancement is in general a powerful tool for isolating the vibrational response of a desired chromophore in complex molecular systems, ${ }^{41}$ in the presence of overlapping ground state absorption and stimulated emission, it is ineffective for discriminating between signals that are originated from ground or excited energy levels. This has so far limited the use of FSRS for mapping excited state properties with respect to its time-domain analogues, namely impulsive stimulated Raman scattering-based techniques, ${ }^{42-47}$ where the coherent oscillations can be directly monitored in the time domain. In particular, phase analysis or the dependence of the time-domain signal on the pulse chirp is a basis for distinguishing between ground or excited electronic state vibrations. ${ }^{48-50}$

In this work, we consider an FSRS experiment in which a highfluence resonant Raman pulse, which, acting also as a photochemical pump, promotes the system ground state population to a desired electronic state prior to the coherent stimulation of the vibrational coherences. Interestingly, FSRS experiments are commonly performed using a positive time delay $T$ between the Raman and the probe pulses (i.e., with the $\mathrm{PP}$ that temporally precedes the RP maximum), to increase both the Raman gain and the spectral resolution. ${ }^{51,52}$ In fact, as depicted in the left panels of Figure 1, because the stimulated vibrational coherences evolve until a second interaction with the $\mathrm{RP}$ that gates the Raman oscillations, using a positive $T$ enables us to increase the effective temporal window in which the Raman coherences are sampled. Under such a condition, only a small portion of the RP precedes the PP and hence most of the molecules are in the ground state at the time of arrival of the probe. Here we demonstrate how the opposite scenario, i.e., a PP temporally following the RP maximum, can be exploited to probe excited state vibrational properties by photoexciting the system in a controlled manner acting on the negative time delay and then stimulating the Raman process because of the joint action of the residual RP with the PP. To evaluate the femtosecond stimulated Raman response under such conditions, we recorded red side FSRS spectra of cresyl violet $(\mathrm{CV}),{ }^{44,53-55}$ an oxazine dye commonly used in histology as stain, characterized by a long-lived excited state, with nonradiative decay to the ground state occurring on the nanosecond time scale. Moreover, CV shows overlapping ground state absorption and stimulated emission, representing hence an excellent candidate for testing the capability of the presented approach for distinguishing between ground and excited state vibrations. CV was dissolved in methanol, and the FSRS spectrum was measured at ambient temperature with the RP tuned to be in resonance with the sample absorption maximum at $\sim 595 \mathrm{~nm}$. A sketch of the two-pulse FSRS experimental setup exploited for the measurements is reported in Figure 1a. Briefly, a Ti:sapphire laser source generates $3.6 \mathrm{~mJ}, 35 \mathrm{fs}$ pulses at 800 $\mathrm{nm}$ and a $1 \mathrm{kHz}$ repetition rate. The synthesis of the $\mathrm{RP}$ is obtained by a two-stage optical parametric amplifier (OPA) that produces tunable near-infrared pulses, followed by a frequency doubling inside a $25 \mathrm{~mm}$ thick beta barium borate (BBO) crystal. Spectral compression inside the thick nonlinear crystal ensures the same time high RP fluences and narrowband pulses. ${ }^{56}$ An additional rectification of the RP temporal and spectral profiles is achieved by a double-pass (2f) spectral filter, ${ }^{57,58}$ which results in Gaussian $\sim 2.5$ ps Raman pulses. The femtosecond PP is a white-light continuum (WLC) obtained by focusing a small portion of the laser fundamental into a sapphire crystal. A variable attenuator and an iris along the beam path before the sapphire crystal are used to tune and optimize the shape of the generated WLC. RP polarization is parallel to the $\mathrm{PP}$, and its fluence is adjusted by using a linear neutral-density 

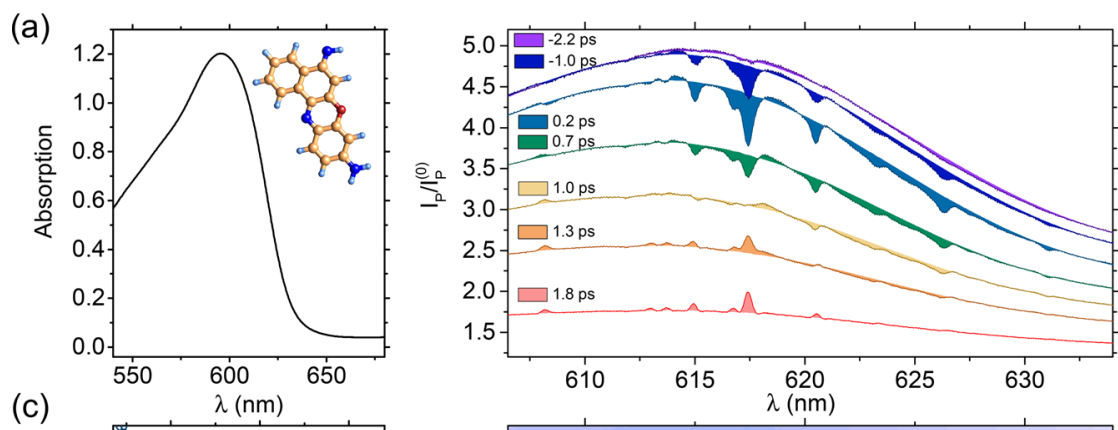

(b)

(c)
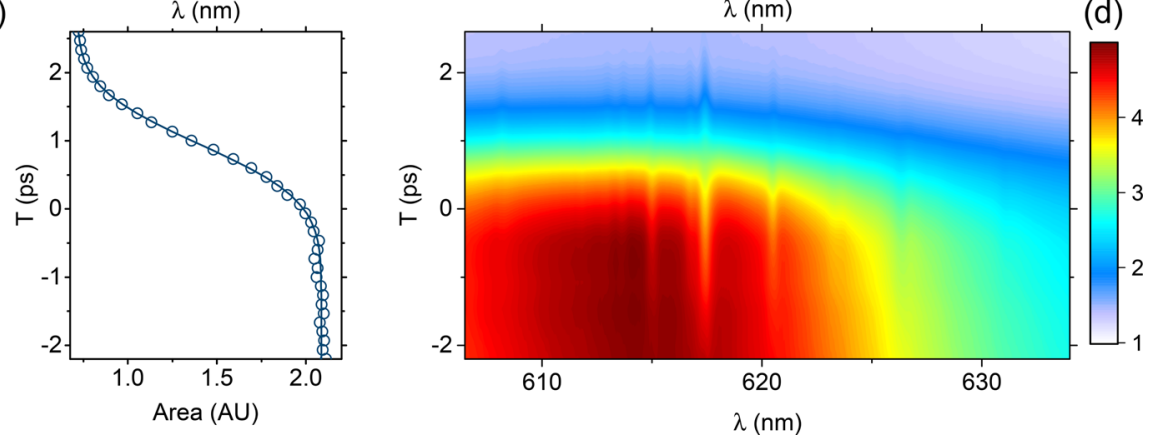

Figure 2. (a) Cresyl violet static absorption spectrum, with a sketch of the sample molecular structure. Due to the RP-induced photoexcitation, FSRS spectra are accompanied by a modification of the PP absorption, which results in a baseline background superimposed on the Raman features. In panel b, we report the FSRS spectrum recorded with a $420 \mathrm{~nJ}$ Raman pump tuned at $\sim 595 \mathrm{~nm}$ before the baseline removal and shown for selected time delays $T$ between the Raman and probe pulses; the filled areas represent the FSRS isolated contributions. The baseline maximum is red-shifted with respect to the maximum absorption due to a small $(\approx 20 \mathrm{~nm})$ Stokes shift. We note that for a PP following the RP the baseline intensity decreases, indicating a reduced quantity of molecules in the electronic excited state (different time delay traces have not been vertically offset). While for positive time delays Raman bands appear as peaks on the top of the baseline background, for negative $T$ values the FSRS spectrum shows negative peaks (losses). In panel d, the corresponding color maps are reported for all of the measured time delays, while in panel c, we show the baseline area as a function of $T$, which can be used to extract a direct estimate of the excited vs ground state molecules.

filer. Further details about the experimental scheme have been described in refs 59 and 60.

The absorption spectrum of the system, together with a sketch of the molecular structure, is reported in Figure 2a, while in Figure $2 \mathrm{~b}$, we report the measured Raman spectra for selected time delays $T$, with the corresponding FSRS color maps for all of the measured $T$ values shown in panel $d$. Notably, the RP photoexcitation induces a modification of the PP transmission, which results in a smooth background due to ground state bleaching and stimulated emission in addition to the FSRS features. The area under such a baseline (shown in Figure 2c) conveniently provides a direct estimate of the $T$-dependent fraction of molecules promoted to the excited electronic state and can be determined by subtracting a polynomial profile obtained as the best fit to the Raman gain in the spectral regions free of Raman bands. As expected, for large positive time delays the baseline vanishes, indicating that all of the molecules probed by FSRS are in the ground state, while for lower $T$ values, the baseline area increases reaching an almost constant value for $T$ values of less than -500 fs. Most importantly, the FSRS line shapes significantly evolve with the delay: the usual positive Raman gains observed for large $T$ values become negative bands with a decrease in $T$ with a trend following the baseline area, suggesting FSRS contributions from excited state vibrational coherences.

To gain further insights into the origin of such negative profiles, we modeled the measured spectra by evaluating the FSRS response trough a perturbative expansion of the density matrix in powers of the $E(t)=\sum_{i} \mathcal{E}_{i}(t) \mathrm{e}^{-i \omega_{i} t}+c$. $c$. electric fields. ${ }^{13,61,62}$ In particular, the Raman gain can be calculated as

$$
\operatorname{RG}(\omega)=-\mathcal{I}\left[\frac{P^{(n)}\left(\omega, T, \lambda_{R}\right)}{\mathcal{E}_{S}^{0}(\omega)}\right]
$$

where $\mathcal{I}(x)$ indicates the imaginary part of $x, \mathcal{E}_{S}^{0}(\omega)$ is the probe field spectral profile (before interaction with the sample), and $P^{(n)}\left(\omega, T, \lambda_{R}\right)$ is the $n$th order total nonlinear polarization induced in the system. ${ }^{63}$ Because several processes, corresponding to different RP and PP field permutations, contribute to the generation of the FSRS signal, several Feynman diagrams, depicting the evolution of the density matrix during consecutive interactions with the electromagnetic fields, have to be considered.

We evaluated the third-order FSRS process with the system initially either in the ground state $(|g\rangle\langle g|)$ or starting from the excited electronic level $(|e\rangle\langle e|)$ upon two preceding interactions with the RP. The initial excited state population, proportional to the measured baseline area, can be directly included in the model from the experimental traces. The Feynman diagrams that take into account the FSRS response are shown in Figure 3. In the top panel, we report the $S_{0}, S_{4}$, and $S_{5}$ processes, where the first $\mathrm{RP}-\mathrm{PP}$ interactions act on the system initially in the electronic ground state $(|g\rangle\langle g|$ population), while in the bottom panel, diagrams $S_{1}-S_{3}$ are associated with processes in which a double interaction with the RP initially promotes the system to the excited electronic state $|e\rangle\langle e|$, which is then interrogated by a subsequent third-order FSRS process. The nonlinear polarization associated with $S_{0}$ can be expressed as 


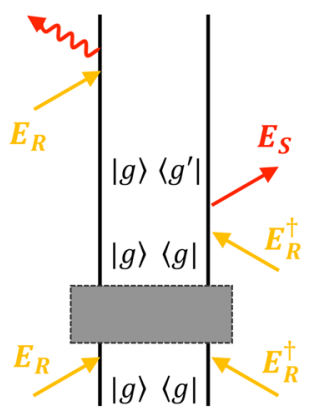

$\mathrm{S}_{0}$

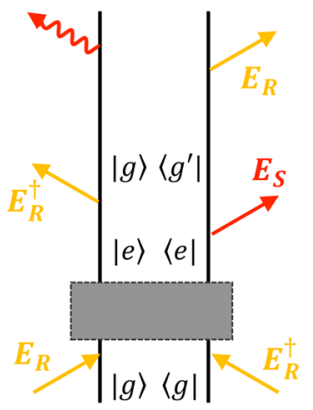

$\mathrm{S}_{1}$

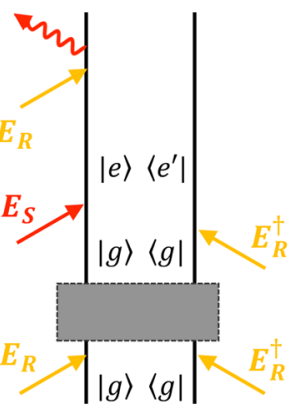

$\mathrm{S}_{4}$

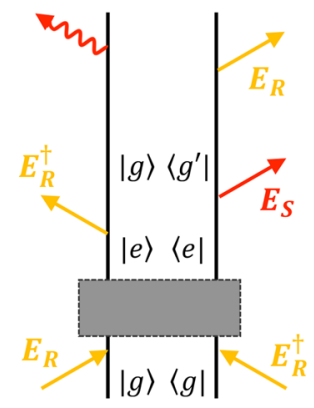

$\mathrm{S}_{2}$

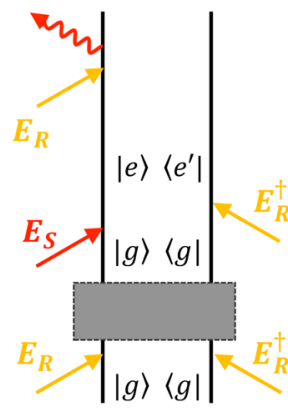

$\mathrm{S}_{5}$
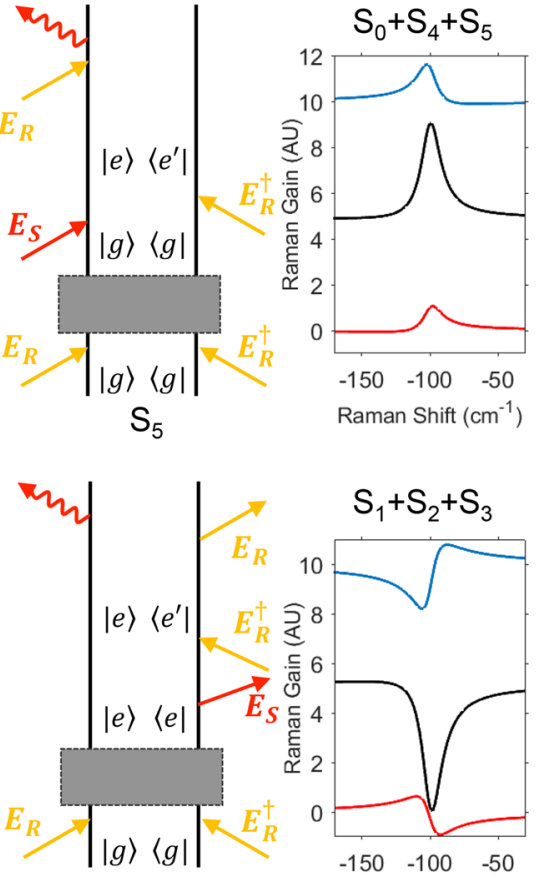

$\mathrm{S}_{3}$

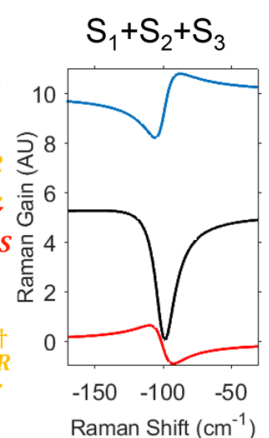

Figure 3. Feynman diagrams describing the FSRS process upon a RP-induced excitation to the $e$ electronic state. Upon a double interaction with the $\mathrm{RP}$, the system can be either in the ground state or in the excited electronic state $(|g\rangle\langle g|$ or $|e\rangle\langle e|$ population, respectively). Upon the joint action of the RP-PP pair, a vibrational coherence, either in the ground state $\left(|g\rangle\left\langle g^{\prime}\right|\right)$ or in the excited electronic stat $\left(|e\rangle\left\langle e^{\prime}\right|\right)$, is stimulated. In the right panels, we evaluate the $S_{0}+S_{4}+5$ and $S_{1}+S_{2}+3$ signals for a model system with a Raman active eigenstate in the $100 \mathrm{~cm}^{-1}$ mode, considering a monochromatic $\mathrm{RP}$ with a wavelength matching the electronic transition and equal dipole moments (black lines): while the system response associated with processes starting from the ground state $\left(S_{0}+S_{4}+5\right.$ diagrams $)$ is a positive gain, diagrams associated with a system initially in the electronic excited state $\left(S_{1}+S_{2}\right.$ $+3)$ result in a negative loss. Red and blue lines show the calculated $S_{0}+S_{4}+5$ and $S_{1}+S_{2}+3$ signals, respectively, obtained by partially detuning the $\mathrm{RP}$ wavelength (considering a $16 \mathrm{~nm}$ shift). The traces corresponding to a different resonance condition have been vertically offset by a constant factor.

$$
\begin{gathered}
P_{S 0}^{(3)}(t, T)=\sum_{g^{\prime} e}\left(\frac{i}{\hbar}\right)^{3} P_{g g}\left|\mu_{g e}\right|^{2}\left|\mu_{e g}\right|^{2} \int_{0}^{\infty} \int_{0}^{\infty} \int_{0}^{\infty} \mathrm{d} \tau_{3} \mathrm{~d} \tau_{2} \mathrm{~d} \tau_{1} \\
\mathcal{E}_{R}^{*}\left(t-\tau_{1}-\tau_{2}-\tau_{3}, T\right) \mathcal{E}_{S}\left(t-\tau_{2}-\tau_{3}\right) \mathcal{E}_{R}\left(t-\tau_{3}, T\right) \\
\mathrm{e}^{i \omega_{R}\left(-\tau_{1}-\tau_{2}\right)} \mathrm{e}^{-i \omega_{S}\left(t-\tau_{2}-\tau_{3}\right)} \mathrm{e}^{-i \widetilde{\omega}_{g e} \tau_{1}} \mathrm{e}^{-i \widetilde{\omega}_{g g}, \tau_{2}} \mathrm{e}^{-i \widetilde{\omega}_{e g}, \tau_{3}}
\end{gathered}
$$

$$
\begin{aligned}
P_{S 1}^{(3)}(\omega, T)= & \sum_{g^{\prime}} \int_{-\infty}^{\infty} \int_{-\infty}^{\infty} \mathrm{d} \omega_{2} \mathrm{~d} \omega_{3} \\
& \frac{\left.P_{e e}\left|\mu_{e g}{ }^{2}\right| \mu_{g e}\right|^{2} \mathcal{E}_{S}\left(\omega-\omega_{S}+\omega_{2}-\omega_{3}\right) \mathcal{E}_{R}^{*}\left(\omega_{2}, T\right) \mathcal{E}_{R}\left(\omega_{3}, T\right)}{\hbar^{3}\left(\omega+\omega_{2}-\omega_{3}-\widetilde{\omega}_{e g}\right)\left(\omega-\omega_{3}-\omega_{R}-\widetilde{\omega}_{g g}\right)\left(\omega-\widetilde{\omega}_{e g}\right)}
\end{aligned}
$$

$$
\begin{aligned}
P_{S 2}^{(3)}(\omega, T)= & \sum_{g^{\prime}} \int_{-\infty}^{\infty} \int_{-\infty}^{\infty} \mathrm{d} \omega_{1} \mathrm{~d} \omega_{3} \\
& \frac{P_{e e}\left|\mu_{e g}\right|^{2} \mid \mu_{g e}{ }^{2} \mathcal{E}_{R}^{*}\left(\omega_{1}, T\right) \mathcal{E}_{S}\left(\omega_{+} \omega_{1}-\omega_{S}-\omega_{3}\right) \mathcal{E}_{R}\left(\omega_{3}, T\right)}{\hbar^{3}\left(\omega_{R}+\omega_{1}+\widetilde{\omega}_{g e}\right)\left(\omega_{R}-\omega+\omega_{3}+\widetilde{\omega}_{g g}\right)\left(\omega-\widetilde{\omega}_{e g}\right)}
\end{aligned}
$$
$\widetilde{\omega}_{i j}=\omega_{i j}-i \gamma_{i j}$. Here, $\omega_{i j}=\omega_{i}-\omega_{j} ; \gamma_{i j}$ indicates the dephasing rate of the $|i\rangle\langle j|$ coherence, $\mu_{i j}$ is the dipole transition moment between states $i$ and $j$, and $P_{g g}$ is the ground state population at the arrival time of the PP. By evaluating the system response in the frequency domain and writing the pulse fields in terms of their Fourier transforms ${ }^{63,64} \mathcal{E}_{S / R}(t)=\int_{-\infty}^{\infty} \mathrm{d} \omega \mathcal{E}_{S / R}(\omega) \mathrm{e}^{-i \omega t}$, we obtain

$$
\begin{aligned}
P_{S 0}^{(3)}(\omega, T)= & -\sum_{g^{\prime} e} \int_{-\infty}^{\infty} \int_{-\infty}^{\infty} \mathrm{d} \omega_{1} \mathrm{~d} \omega_{3} \\
& \frac{P_{g g}\left|\mu_{g e}\right|^{2}\left|\mu_{e g}\right|^{2} \mathcal{E}_{R}^{*}\left(\omega_{1}, T\right) \mathcal{E}_{S}\left(\omega+\omega_{1}-\omega_{S}-\omega_{3}\right) \mathcal{E}_{R}\left(\omega_{3}, T\right)}{\hbar^{3}\left(\omega_{R}+\omega_{1}+\widetilde{\omega}_{g e}\right)\left(\omega_{R}-\omega+\omega_{3}+\widetilde{\omega}_{g g}\right)\left(\omega-\widetilde{\omega}_{e g}\right)}
\end{aligned}
$$

Similarly, the other nonlinear polarization terms associated with diagrams $S_{1}-S_{5}$ can be obtained and expressed as

$$
\begin{aligned}
P_{S 3}^{(3)}(\omega, T)= & \sum_{e^{\prime} g^{\prime}} \int_{-\infty}^{\infty} \int_{-\infty}^{\infty} \mathrm{d} \omega_{2} \mathrm{~d} \omega_{3} \\
& \frac{P_{e e}\left|\mu_{g^{\prime} e}\right|^{2}\left|\mu_{g^{\prime} e}\right|^{2} \mathcal{E}_{S}\left(\omega-\omega_{S}+\omega_{2}-\omega_{3}\right) \mathcal{E}_{R}^{*}\left(\omega_{2}, T\right) \mathcal{E}_{R}\left(\omega_{3}, T\right)}{\hbar^{3}\left(\omega+\omega_{2}-\omega_{3}-\widetilde{\omega}_{e g^{\prime}}\right)\left(\omega-\omega_{3}-\omega_{R}-\widetilde{\omega}_{e e}\right)\left(\omega-\widetilde{\omega}_{e g^{\prime}}\right)}
\end{aligned}
$$

$$
\begin{aligned}
P_{S 4}^{(3)}(\omega, T)= & -\sum_{e^{\prime}} \int_{-\infty}^{\infty} \int_{-\infty}^{\infty} \mathrm{d} \omega_{1} \mathrm{~d} \omega_{3} \\
& \frac{P_{e e}\left|\mu_{g e \prime}\right|^{2}\left|\mu_{g e}\right|^{2} \mathcal{E}_{R}^{*}\left(\omega_{1}, T\right) \mathcal{E}_{S}\left(\omega+\omega_{1}-\omega_{S}-\omega_{3}\right) \mathcal{E}_{R}\left(\omega_{3}, T\right)}{\hbar^{3}\left(\omega_{R}+\omega_{1}+\widetilde{\omega}_{g e}\right)\left(\omega_{R}-\omega+\omega_{3}+\widetilde{\omega}_{e e}\right)\left(\omega-\widetilde{\omega}_{e g}\right)}
\end{aligned}
$$

$$
P_{S S}^{(3)}(\omega, T)=-\sum_{e^{\prime}} \int_{-\infty}^{\infty} \int_{-\infty}^{\infty} \mathrm{d} \omega_{2} \mathrm{~d} \omega_{3}
$$$$
\frac{P_{g g}\left|\mu_{g e l}\right|^{2}\left|\mu_{g e}\right|^{2} \mathcal{E}_{S}\left(\omega-\omega_{S}+\omega_{2}-\omega_{3}\right) \mathcal{E}_{R}^{*}\left(\omega_{2}, T\right) \mathcal{E}_{R}\left(\omega_{3}, T\right)}{\hbar^{3}\left(\omega+\omega_{2}-\omega_{3}-\widetilde{\omega}_{e g}\right)\left(\omega-\omega_{3}-\omega_{R}-\widetilde{\omega}_{e e}\right)\left(\omega-\widetilde{\omega}_{e g}\right)}
$$ 

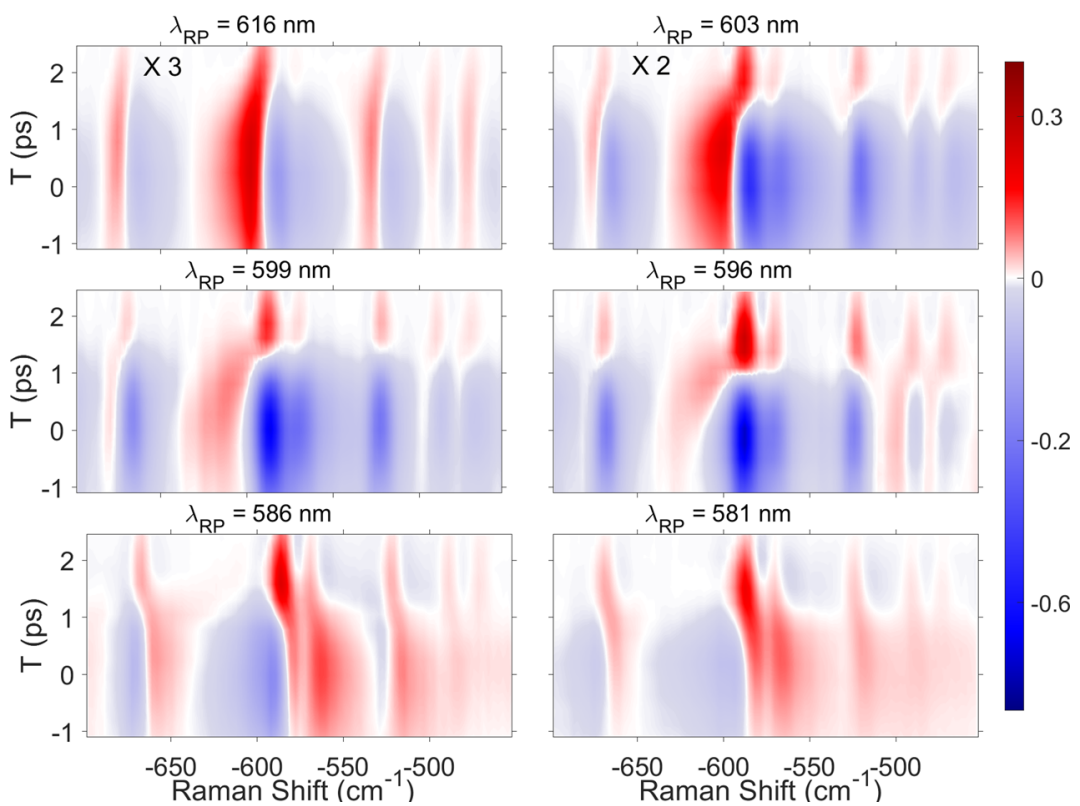

Figure 4. RP wavelength dependence of the FSRS spectra as a function of wavenumber and relative time delay between RP and PP. While for positive time delays, where the signal originated from processes starting from the ground state, only positive Raman gains are recorded, for negative $T$ values we observe a signal profile that depends on the RP wavelength, as expected in the presence of a signal involving excited electronic state excitations.
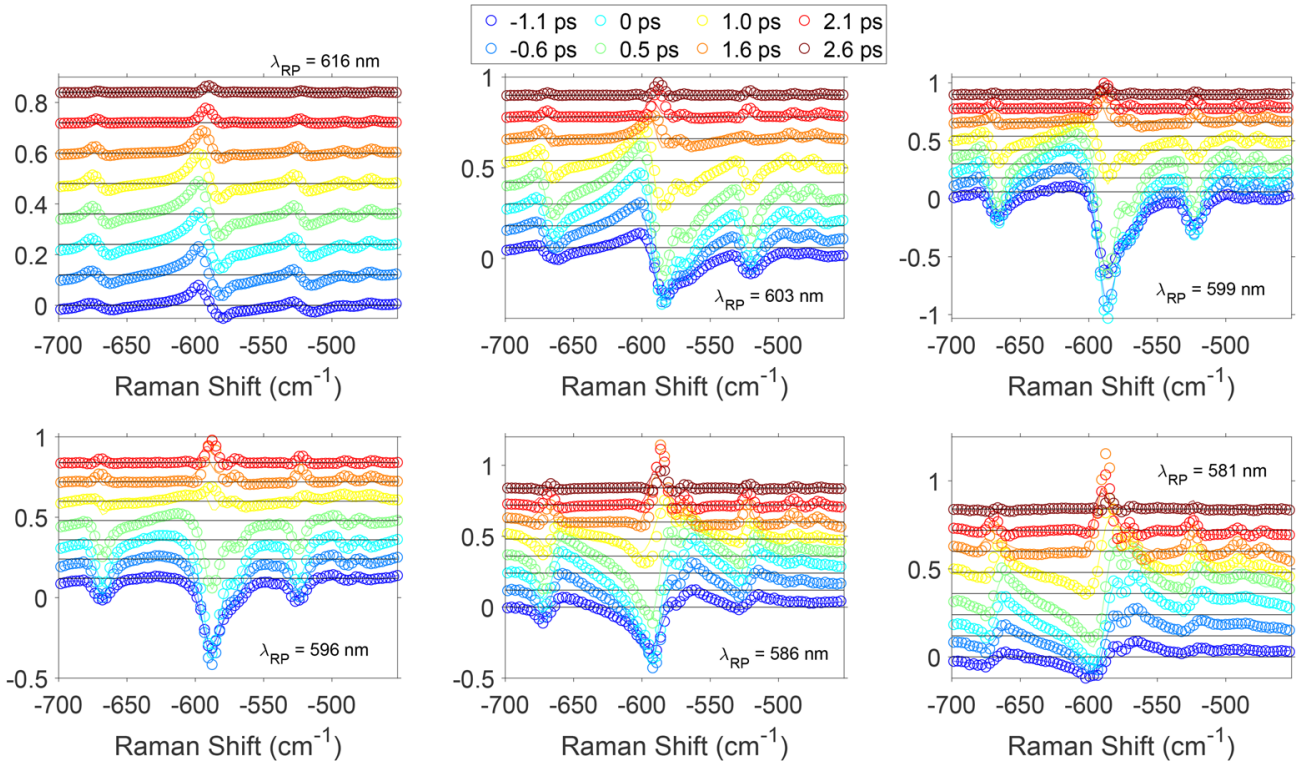

Figure 5. Wavelength dependence of the FSRS spectra. The data (circles) and model (solid lines) are compared as a function of RP wavelength $\lambda_{\mathrm{RP}}$ and relative time delay $T$ between the Raman and probe pulses. For a RP tuned to be in resonance with a system electronic transition, Raman excitation processes arising from the system initially in the excited state result in intense negative losses (negative time delays, with a RP at 596-599 nm). Tuning the RP away from a full resonant condition turns the negative signal into a dispersive profile, with an odd symmetry: with a blue-shift in the RP the positive lobe is at higher wavenumbers, and a red-shift of the RP generates a positive lobe at lower frequencies. Traces acquired at different $T$ values have been vertically offset by a constant factor.

where $P_{e e}$ indicates the electronic excited state population at the arrival time of the PP. A complete derivation of the $S_{0}-S_{5}$ signals is reported in the Supporting Information. We note that to correctly evaluate the total system response in the presence of a resonant RP, diagrams $S_{4}$ and $S_{5}$, which consider a system initially in the ground state, with a vibrational coherence $|e\rangle\left\langle e^{\prime}\right|$ generated upon the joint action of the RP-PP pair, should be considered. Interestingly, as clarified in the Supporting Information, for an off-resonant Raman pulse, the $S_{4}$ and $S_{5}$ responses result in a Lorentzian response with opposite sign and hence cancel out.

In the right panel of Figure 3, we show the corresponding $S_{0}-$ $S_{5}$ signals, with $S_{j}=-\mathcal{I}\left[\frac{P_{S_{j}}^{(3)}(\omega)}{\mathcal{E}_{S}^{0}(\omega)}\right]$, evaluated considering for the sake of simplicity equal weights and dipole moments for all of the diagrams: for a perfectly resonant RP, while the system response associated with processes starting from $|g\rangle\langle g|$ is a positive gain, diagrams in which the system is initially in an excited electronic state population result in a negative loss. 


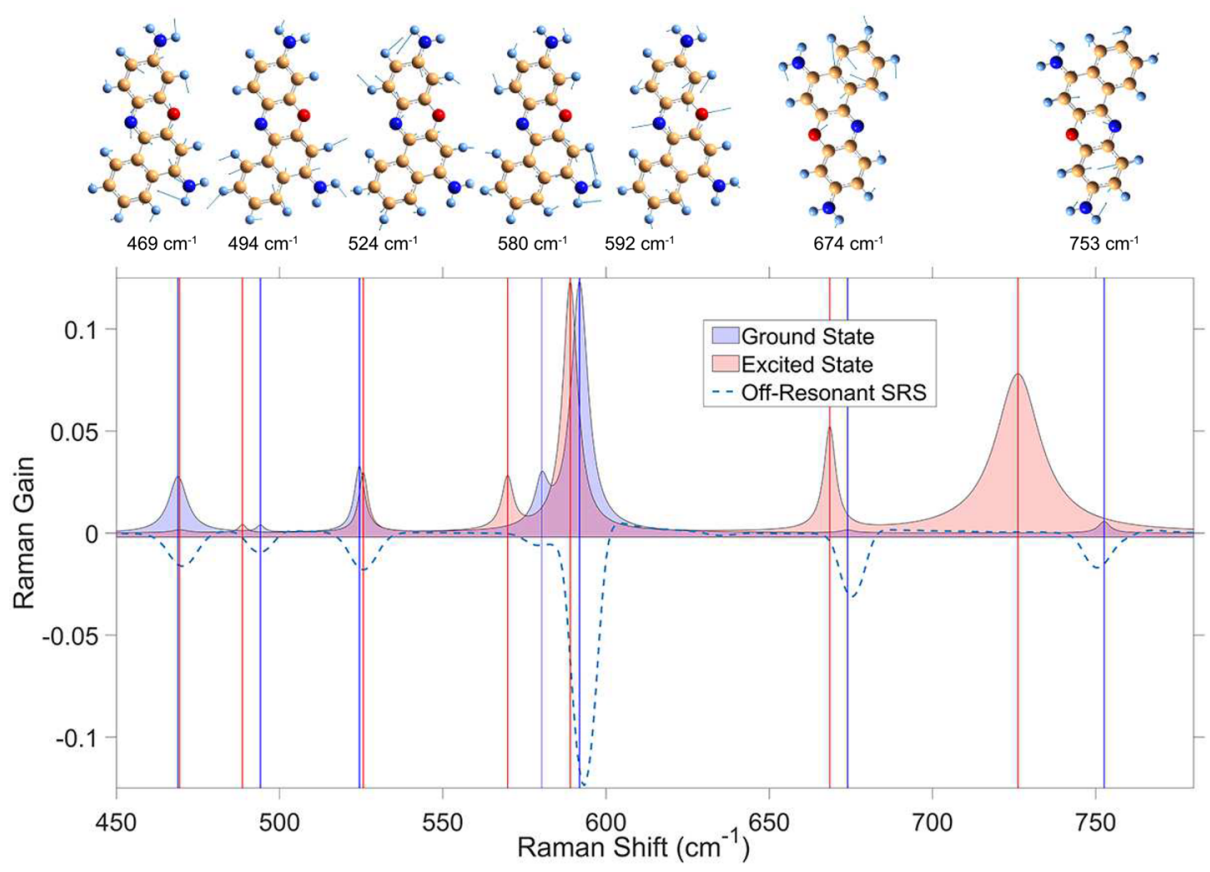

Figure 6. Comparison between the nonresonant FSRS spectrum recorded in the blue side (dashed line) and the extracted resonant Raman profiles for ground and excited electronic state coherences (blue and red filled areas, respectively), with the Raman gains that have been normalized at the intensity of the stronger band $\left(\right.$ at $\left.\approx 590 \mathrm{~cm}^{-1}\right)$. Vertical blue and red lines indicate the extracted Raman mode frequencies in the ground and excited states, respectively. Extracted and measured ground state peak central positions are in agreement, while the different Raman intensities are expected in view of the resonant vs off-resonant conditions. The presence of purely negative peaks ensures the complete off-resonant condition for the blue side FSRS spectrum. ${ }^{39}$ In the top panel, sketches of the ground state elongation eigenvectors computed by density functional theory with the B3LYP functional and the $6-311++\mathrm{G}(\mathrm{d}, \mathrm{p})$ basis set are shown. ${ }^{65,66}$

Notably, detuning the RP away from a perfectly resonant condition has almost no effect on the Raman line shape of the $S_{0}$, $S_{4}$, and $S_{5}$ diagrams and reduces only their corresponding signal intensity. On the contrary, it results in a dispersive profile for $S_{1}-$ $S_{3}$, i.e., for those pathways starting from the excited $|e\rangle\langle e|$ state. As shown in the right panel of Figure 3, for a RP red-shifted with respect to the maximum absorption, the positive lobe of such a dispersive profile is at lower frequencies (larger absolute Raman shift), while blue-shifting the RP generates a positive lobe at lower frequencies. Interestingly, for the $S_{3}$ diagram, which involves the creation of excited state vibrational coherences, the first three interactions occur on the bra side of the density matrix, resulting in a mode specific resonant enhancement condition similar to the one characterizing the ground state blue side SRS response. ${ }^{39}$ In fact, the first and third denominators in eq 6 give rise to a resonant enhancement for a RP wavelength matching the energy difference $\hbar\left(\omega_{e g}+\omega_{e^{\prime} e}\right)$. To verify the presence of such dispersive profiles in the measured spectra and hence also the presence of $|e\rangle\langle e|$ excitations, we recorded cresyl violet FSRS spectra scanning the RP wavelength across the resonance profile. In Figure 4, we report the corresponding color map as a function of Raman shift and temporal delay $T$ between the RP-PP pair. Slices for selected time delays are also reported in Figure 5 as colored circles. As expected, while for a high positive $T$ the RP-PP interaction stimulating the vibrational coherences acts on only those molecules initially in the ground state and generates only positive Raman gains, for negative $T$ values we observe negative or dispersive line shapes indicating the presence of excited state Raman coherences. We note that also for some positive time delays $(T<1 \mathrm{ps})$, a significant fraction of molecules is in the excited state at the arrival time of the probe.
To retrieve the vibrational information associated with the measured FSRS spectra, we performed a global fit on the experimental traces using eqs $1-8$. The Raman pulse $\mathcal{E}_{R}(t)$ and the probe pulse $\mathcal{E}_{S}(t)$ temporal envelopes have been modeled as Gaussian profiles:

$$
\begin{aligned}
& \mathcal{E}_{R}(t)=\mathcal{E}_{R}^{0} \mathrm{e}^{-(t-T)^{2} / 2 \sigma_{R}^{2}} \mathrm{e}^{-i \omega_{R} t} \\
& \mathcal{E}_{S}(t)=\mathcal{E}_{S}^{0} \mathrm{e}^{-t^{2} / 2 \sigma_{P}^{2}} \mathrm{e}^{-i \omega_{S} t}
\end{aligned}
$$

where the parameters $\omega_{b a}$ and $\sigma_{R}$ have been adjusted to best fit the experimental data $\left(\omega_{b a}=16900 \mathrm{~cm}^{-1}\right.$, the electronic dephasing rate $\gamma_{b a}=580 \mathrm{~cm}^{-1}$, with an extracted RP duration equal to $\approx 2.5$ ps in agreement with the experimental one), while the initial ground and excited state populations $P_{g g}$ and $P_{e e}$ respectively, have been extracted from the baseline area. In Figure 5, the experimental FSRS spectra (colored circles) are compared with the simulations (solid lines), showing a good agreement. This is further corroborated by the comparison between the extracted ground and excited electronic state FSRS spectrum and the nonresonant blue side stimulated Raman spectrum reported in Figure 6, with peak positions and amplitudes reported in Table 1 . As expected, the extracted electronic ground state Raman line positions are in line with the blue side Raman losses, validating the capability of assigning the measured vibrations to the corresponding electronic state. Notably, the excited state normal modes show in general small frequency decreases indicating reduced excited energy level force constants (with respect to the ground state potentials). For the 590, 669, and $726 \mathrm{~cm}^{-1}$ Raman modes, we identify high transition dipole moments $\mu_{g e^{\prime}}$, pointing to the reaction coordinate nature of such Raman excitations. ${ }^{12,13}$ 
Table 1. Ground and Excited State Peak Positions and Intensities

\begin{tabular}{cccc}
$\tilde{\nu}_{g^{\prime} g}\left(\mathrm{~cm}^{-1}\right)$ & $\tilde{\nu}_{e^{\prime} e}\left(\mathrm{~cm}^{-1}\right)$ & $\left|\mu_{e g^{\prime}}\right|^{2}(\mathrm{AU})$ & $\left|\mu_{g e^{\prime}}\right|^{2}(\mathrm{AU})$ \\
469 & 469 & 0.7 & 0.1 \\
494 & 489 & 0.1 & 0.3 \\
524 & 526 & 0.9 & 2.4 \\
580 & 570 & 0.6 & 2.1 \\
592 & 589 & 3.3 & 10 \\
674 & 669 & 0.04 & 4.2 \\
753 & 726 & 0.15 & 6.5 \\
\hline
\end{tabular}

It is worth stressing that, because several processes contribute to the generation of the measured signal and a small detuning of the RP from a perfectly resonant condition results in disperive Raman line shapes, it is crucial to build on eqs $1-8$ for correctly extracting the exact Raman excitation frequencies and line widths. This procedure establishes a convenient protocol for measuring and assigning vibrational properties to a targeted excited electronic state, and it is particularly convenient for the challenging case of overlapping ground state absorption and stimulated emission from the excited electronic state, when the conventional resonance enhancement is not effective for discriminating between signals that originated from different potential energy surfaces.

These results also indicate that large Raman pump fluences do not help, in general, in recording more accurate ground state FSRS spectra. Despite the linear dependence of the Raman cross section on the RP intensity, indeed, increasing the RP energy can promote the system to the excited state. Accordingly, concurring signals are generated with a dispersive profile or even a negative sign, overwhelming the desired Raman information. This result rationalizes also why, under resonance conditions, the FSRS spectrum measured in the blue side can show more intense Raman bands, providing a better signal-to-noise ratio. In fact, while in the blue side the resonance condition is red-shifted with respect to the electronic absorption peak ${ }^{39}$ and hence the promotion of the system to the excited state is avoided, in the red side the resonance enhancement is achieved for a Raman wavelength that matches the electronic transition and that can promote the system to the excited state, with the signals that originated from excited state coherences that can destructively interfere with the ground state FSRS response.

In summary, we have investigated the FSRS response in the presence of a RP photoinduced excited state population. A diagrammatic treatment of the signal generation has been exploited to analyze the data, scrutinizing the concurring pathways that generate the nonlinear Raman response and discriminating between processes involving ground and excited state coherences. We have shown how to experimentally control the excited state population tuning the relative time delay between Raman and probe pulses, and by comparing the FSRS response as a function of the Raman resonance condition, we have demonstrated how to extract the excited state vibrational line shapes, developing a novel FSRS protocol for recording excited state Raman spectra. As a benchmark of the proposed experimental scheme and theoretical model, we applied the technique to study cresyl violet, dissecting its ground and excited state vibrational properties and identifying reduced force constants in the excited state. We anticipate that the approach presented here holds the potential for selectively mapping the reaction coordinates pertaining to different electronic stages implied in photoactive compounds.

\section{ASSOCIATED CONTENT}

\section{Supporting Information}

The Supporting Information is available free of charge at https://pubs.acs.org/doi/10.1021/acs.jpclett.0c01971.

Supporting material with the complete derivation of the $S_{0}-S_{5}$ signals (PDF)

\section{AUTHOR INFORMATION}

\section{Corresponding Author}

Tullio Scopigno - Dipartimento di Fisica, Universitá di Roma "La Sapienza", Roma I-00185, Italy; Center for Life Nano Science@Sapienza and Graphene Labs, Istituto Italiano di Tecnologia, Roma I-00161, Italy; (1) orcid.org/0000-00027437-4262; Email: tullio.scopigno@romal.infn.it

\section{Authors}

Giovanni Batignani - Dipartimento di Fisica, Universitá di Roma "La Sapienza", Roma I-00185, Italy; 이이.org/00000002-6214-8604

Carino Ferrante - Dipartimento di Fisica, Universitá di Roma "La Sapienza", Roma I-00185, Italy; Center for Life Nano Science@Sapienza and Graphene Labs, Istituto Italiano di Tecnologia, Roma I-00161, Italy; 이이.org/0000-00026391-0672

Complete contact information is available at:

https://pubs.acs.org/10.1021/acs.jpclett.0c01971

\section{Notes}

The authors declare no competing financial interest.

\section{ACKNOWLEDGMENTS}

G.B. and T.S. acknowledge the 'Progetti di Ricerca Medi 2019' grant by Sapienza Universitá di Roma. This project has received funding from the PRIN 2017 Project, Grant No. 201795SBA3HARVEST, and from the European Union's Horizon 2020 research and innovation program Graphene Flagship under Grant Agreement No. 881603.

\section{REFERENCES}

(1) Zewail, A. H. Femtochemistry: Atomic-Scale Dynamics of the Chemical Bond. J. Phys. Chem. A 2000, 104, 5660-5694.

(2) Potma, E. O.; Mukamel, S. In Coherent Raman Scattering Microscopy; Cheng, J.-X., Xie, X. S., Eds.; CRC Press: Boca Raton, FL, 2012.

(3) Bardeen, C. J.; Wang, Q.; Shank, C. V. Selective Excitation of Vibrational Wave Packet Motion Using Chirped Pulses. Phys. Rev. Lett. 1995, 75, 3410-3413.

(4) Wand, A.; Kallush, S.; Shoshanim, O.; Bismuth, O.; Kosloff, R.; Ruhman, S. Chirp Effects on Impulsive Vibrational Spectroscopy: a Multimode Perspective. Phys. Chem. Chem. Phys. 2010, 12, 2149-2163.

(5) Biggs, J. D.; Zhang, Y.; Healion, D.; Mukamel, S. Multidimensional $\mathrm{X}$-Ray Spectroscopy of Valence and Core Excitations in Cysteine. J. Chem. Phys. 2013, 138, 144303.

(6) Wende, T.; Liebel, M.; Schnedermann, C.; Pethick, R. J.; Kukura, P. Population-Controlled Impulsive Vibrational Spectroscopy: Background- and Baseline-Free Raman Spectroscopy of Excited Electronic States. J. Phys. Chem. A 2014, 118, 9976-9984.

(7) Dorfman, K. E.; Bennett, K.; Mukamel, S. Detecting Electronic Coherence by Multidimensional Broadband Stimulated X-Ray Raman Signals. Phys. Rev. A: At., Mol., Opt. Phys. 2015, 92, 023826.

(8) Dorfman, K. E.; Schlawin, F.; Mukamel, S. Nonlinear Optical Signals and Spectroscopy with Quantum Light. Rev. Mod. Phys. 2016, 88,045008 . 
(9) Versteeg, R. B.; Zhu, J.; Padmanabhan, P.; Boguschewski, C.; German, R.; Goedecke, M.; Becker, P.; van Loosdrecht, P. H. M. A Tunable Time-Resolved Spontaneous Raman Spectroscopy Setup for Probing Ultrafast Collective Excitation and Quasiparticle Dynamics in Quantum Materials. Struct. Dyn. 2018, 5, 044301.

(10) Maiuri, M.; Ostroumov, E. E.; Saer, R. G.; Blankenship, R. E.; Scholes, G. D. Coherent wavepackets in the Fenna-Matthews-Olson complex are robust to excitonic-structure perturbations caused by mutagenesis. Nat. Chem. 2018, 10, 177-183.

(11) Kuramochi, H.; Takeuchi, S.; Kamikubo, H.; Kataoka, M.; Tahara, T. Fifth-order time-domain Raman spectroscopy of photoactive yellow protein for visualizing vibrational coupling in its excited state. Sci. Adv. 2019, 5, eaau4490.

(12) Fumero, G.; Schnedermann, C.; Batignani, G.; Wende, T.; Liebel, M.; Bassolino, G.; Ferrante, C.; Mukamel, S.; Kukura, P.; Scopigno, T. Two-Dimensional Impulsively Stimulated Resonant Raman Spectroscopy of Molecular Excited States. Phys. Rev. X 2020, 10,011051 .

(13) Mukamel, S. Principles of Nonlinear Spectroscopy; Oxford University Press: New York, 1995.

(14) Freudiger, C. W.; Min, W.; Saar, B. G.; Lu, S.; Holtom, G. R.; He, C.; Tsai, J. C.; Kang, J. X.; Xie, X. S. Label-Free Biomedical Imaging with High Sensitivity by Stimulated Raman Scattering Microscopy. Science 2008, 322, 1857-1861.

(15) Zong, C.; Premasiri, R.; Lin, H.; Huang, Y.; Zhang, C.; Yang, C.; Ren, B.; Ziegler, L. D.; Cheng, J.-X. Plasmon-enhanced stimulated Raman scattering microscopy with single-molecule detection sensitivity. Nat. Commun. 2019, 10, 5318.

(16) De la Cadena, A.; Valensise, C. M.; Marangoni, M.; Cerullo, G.; Polli, D. Broadband stimulated Raman scattering microscopy with wavelength-scanning detection. J. Raman Spectrosc. 2020, DOI: $10.1002 /$ jrs.5816.

(17) Nestor, J.; Spiro, T. G.; Klauminzer, G. Coherent anti-Stokes Raman scattering (CARS) spectra, with resonance enhancement, of cytochrome $\mathrm{c}$ and vitamin B12 in dilute aqueous solution. Proc. Natl. Acad. Sci. U. S. A. 1976, 73, 3329-3332.

(18) Virga, A.; Ferrante, C.; Batignani, G.; De Fazio, D.; Nunn, A. D. G.; Ferrari, A. C.; Cerullo, G.; Scopigno, T. Coherent anti-Stokes Raman spectroscopy of single and multi-layer graphene. Nat. Commun. 2019, 10, 3658.

(19) Mukamel, S.; Biggs, J. D. Communication: Comment on the effective temporal and spectral resolution of impulsive stimulated Raman signals. J. Chem. Phys. 2011, 134, 161101.

(20) Fumero, G.; Batignani, G.; Dorfman, K. E.; Mukamel, S.; Scopigno, T. On the Resolution Limit of Femtosecond Stimulated Raman Spectroscopy: Modelling Fifth-Order Signals with Overlapping Pulses. ChemPhysChem 2015, 16, 3438-3443.

(21) Yoshizawa, M.; Hattori, Y.; Kobayashi, T. Femtosecond timeresolved resonance Raman gain spectroscopy in polydiacetylene. Phys. Rev. B: Condens. Matter Mater. Phys. 1994, 49, 13259.

(22) Yoshizawa, M.; Kubo, M.; Kurosawa, M. Ultrafast photoisomerization in DCM dye observed by new femtosecond Raman spectroscopy. J. Lumin. 2000, 87, 739.

(23) Kukura, P.; McCamant, D. W.; Mathies, R. A. Femtosecond Stimulated Raman Spectroscopy. Annu. Rev. Phys. Chem. 2007, 58, 461.

(24) Kukura, P.; McCamant, D. W.; Yoon, S.; Wandschneider, D. B.; Mathies, R. A. Structural Observation of the Primary Isomerization in Vision with Femtosecond-Stimulated Raman. Science 2005, 310, 10061009.

(25) Laimgruber, S.; Schachenmayr, H.; Schmidt, B.; Zinth, W.; Gilch, P. A femtosecond stimulated raman spectrograph for the near ultraviolet. Appl. Phys. B: Lasers Opt. 2006, 85, 557-564.

(26) Fang, C.; Frontiera, R. R.; Tran, R.; Mathies, R. A. Mapping GFP structure evolution during proton transfer with femtosecond Raman spectroscopy. Nature 2009, 462, 200.

(27) Provencher, F.; Bérubé, N.; Parker, A. W.; Greetham, G. M.; Towrie, M.; Hellmann, C.; Côté, M.; Stingelin, N.; Silva, C.; Hayes, S. C. Direct observation of ultrafast long-range charge separation at polymer-fullerene heterojunctions. Nat. Commun. 2014, 5, 4288.
(28) Zhou, J.; Yu, W.; Bragg, A. E. Structural Relaxation of Photoexcited Quaterthiophenes Probed with Vibrational Specificity. J. Phys. Chem. Lett. 2015, 6, 3496.

(29) Batignani, G.; Pontecorvo, E.; Ferrante, C.; Aschi, M.; Elles, C. G.; Scopigno, T. Visualizing Excited-State Dynamics of a Diaryl Thiophene: Femtosecond Stimulated Raman Scattering as a Probe of Conjugated Molecules. J. Phys. Chem. Lett. 2016, 7, 2981-2988.

(30) Ferrante, C.; Pontecorvo, E.; Cerullo, G.; Vos, M. H.; Scopigno, T. Direct observation of subpicosecond vibrational dynamics in photoexcited myoglobin. Nat. Chem. 2016, 8, 1137-1143.

(31) Barclay, M. S.; Quincy, T. J.; Williams-Young, D. B.; Caricato, M.; Elles, C. G. Accurate Assignments of Excited-State Resonance Raman Spectra: A Benchmark Study Combining Experiment and Theory. J. Phys. Chem. A 2017, 121, 7937-7946.

(32) Hall, C. R.; Conyard, J.; Heisler, I. A.; Jones, G.; Frost, J.; Browne, W. R.; Feringa, B. L.; Meech, S. R. Ultrafast Dynamics in Light-Driven Molecular Rotary Motors Probed by Femtosecond Stimulated Raman Spectroscopy. J. Am. Chem. Soc. 2017, 139, 7408-7414.

(33) Piontkowski, Z.; McCamant, D. W. Excited-State Planarization in Donor-Bridge Dye Sensitizers: Phenylene versus Thiophene Bridges. J. Am. Chem. Soc. 2018, 140, 11046-11057.

(34) Quincy, T. J.; Barclay, M. S.; Caricato, M.; Elles, C. G. Probing Dynamics in Higher-Lying Electronic States with Resonance-Enhanced Femtosecond Stimulated Raman Spectroscopy. J. Phys. Chem. A 2018, $122,8308-8319$.

(35) Barclay, M. S.; Caricato, M.; Elles, C. G. Femtosecond Stimulated Raman Scattering from Triplet Electronic States: Experimental and Theoretical Study of Resonance Enhancements. J. Phys. Chem. A 2019, 123, 7720-7732.

(36) Batignani, G.; Pontecorvo, E.; Bossini, D.; Ferrante, C.; Fumero, G.; Cerullo, G.; Mukamel, S.; Scopigno, T. Modeling the Ultrafast Response of Two-Magnon Raman Excitations in Antiferromagnets on the Femtosecond Timescale. Ann. Phys. 2019, 531, 1900439.

(37) Sotome, H.; Une, K.; Nagasaka, T.; Kobatake, S.; Irie, M.; Miyasaka, H. A dominant factor of the cycloreversion reactivity of diarylethene derivatives as revealed by femtosecond time-resolved absorption spectroscopy. J. Chem. Phys. 2020, 152, 034301.

(38) Prince, R. C.; Frontiera, R. R.; Potma, E. O. Stimulated Raman Scattering: From Bulk to. Chem. Rev. 2017, 117, 5070-5094.

(39) Batignani, G.; Pontecorvo, E.; Giovannetti, G.; Ferrante, C.; Fumero, G.; Scopigno, T. Electronic resonances in broadband stimulated Raman spectroscopy. Sci. Rep. 2016, 6, 18445.

(40) Harbola, U.; Umapathy, S.; Mukamel, S. Loss and gain signals in broadband stimulated-Raman spectra: Theoretical analysis. Phys. Rev. A: At., Mol., Opt. Phys. 2013, 88, No. 011801(R).

(41) Spiro, T. G. Biological Applications of Resonance Raman Spectroscopy: Haem Proteins. Proc. R. Soc. A 1975, 345, 89-105.

(42) Liebel, M.; Kukura, P. Broad-Band Impulsive Vibrational Spectroscopy of Excited Electronic States in the Time Domain. J. Phys. Chem. Lett. 2013, 4, 1358-1364.

(43) Fujisawa, T.; Kuramochi, H.; Hosoi, H.; Takeuchi, S.; Tahara, T. Role of Coherent Low-Frequency Motion in Excited-State Proton Transfer of Green Fluorescent Protein Studied by Time-Resolved Impulsive Stimulated Raman Spectroscopy. J. Am. Chem. Soc. 2016, $138,3942-3945$.

(44) Rafiq, S.; Scholes, G. D. Slow Intramolecular Vibrational Relaxation Leads to Long-Lived Excited-State Wavepackets. J. Phys. Chem. A 2016, 120, 6792-6799.

(45) Park, M.; Neukirch, A. J.; Reyes-Lillo, S. E.; Lai, M.; Ellis, S. R.; Dietze, D.; Neaton, J. B.; Yang, P.; Tretiak, S.; Mathies, R. A. Excitedstate vibrational dynamics toward the polaron in methylammonium lead iodide perovskite. Nat. Commun. 2018, 9, 2525.

(46) Kuramochi, H.; Takeuchi, S.; Iwamura, M.; Nozaki, K.; Tahara, $\mathrm{T}$. Tracking Photoinduced $\mathrm{Au}-\mathrm{Au}$ Bond Formation through Transient Terahertz Vibrations Observed by Femtosecond Time-Domain Raman Spectroscopy. J. Am. Chem. Soc. 2019, 141, 19296-19303.

(47) Kim, W.; Kim, T.; Kang, S.; Hong, Y.; Würthner, F.; Kim, D. Tracking Structural Evolution during Symmetry Breaking Charge Separation in Quadrupolar Perylene Bisimide with Time-Resolved 
Impulsive Stimulated Raman Spectroscopy. Angew. Chem. 2020, 132, 8382.

(48) Zeiger, H. J.; Vidal, J.; Cheng, T. K.; Ippen, E. P.; Dresselhaus, G.; Dresselhaus, M. S. Theory for displacive excitation of coherent phonons. Phys. Rev. B: Condens. Matter Mater. Phys. 1992, 45, 768-778.

(49) Monacelli, L.; Batignani, G.; Fumero, G.; Ferrante, C.; Mukamel, S.; Scopigno, T. Manipulating Impulsive Stimulated Raman Spectroscopy with a Chirped Probe Pulse. J. Phys. Chem. Lett. 2017, 8, 966-974.

(50) Batignani, G.; Fumero, G.; Srimath Kandada, A. R.; Cerullo, G.; Gandini, M.; Ferrante, C.; Petrozza, A.; Scopigno, T. Probing femtosecond lattice displacement upon photo-carrier generation in lead halide perovskite. Nat. Commun. 2018, 9, 1971.

(51) Yoon, S.; McCamant, D. W.; Kukura, P.; Mathies, R. A.; Zhang, D.; Lee, S.-Y. Dependence of line shapes in femtosecond broadband stimulated Raman spectroscopy on pump-probe time delay. J. Chem. Phys. 2005, 122, 024505.

(52) Ferrante, C.; Batignani, G.; Fumero, G.; Pontecorvo, E.; Virga, A.; Montemiglio, L. C.; Cerullo, G.; Vos, M. H.; Scopigno, T. Resonant broadband stimulated Raman scattering in myoglobin. J. Raman Spectrosc. 2018, 49, 913-920.

(53) Isak, S. J.; Eyring, E. M. Fluorescence quantum yield of cresyl violet in methanol and water as a function of concentration. J. Phys. Chem. 1992, 96, 1738-1742.

(54) Vogel, E.; Gbureck, A.; Kiefer, W. Vibrational spectroscopic studies on the dyes cresyl violet and coumarin 152. J. Mol. Struct. 2000, 550-551, 177-190.

(55) Brazard, J.; Bizimana, L. A.; Gellen, T.; Carbery, W. P.; Turner, D. B. Experimental Detection of Branching at a Conical Intersection in a Highly Fluorescent Molecule. J. Phys. Chem. Lett. 2016, 7, 14-19.

(56) Marangoni, M.; Brida, D.; Conforti, M.; Capobianco, A. D.; Manzoni, C.; Baronio, F.; Nalesso, G. F.; De Angelis, C.; Ramponi, R.; Cerullo, G. Synthesis of picosecond pulses by spectral compression and shaping of femtosecond pulses in engineered quadratic nonlinear media. Opt. Lett. 2009, 34, 241.

(57) Pontecorvo, E.; Ferrante, C.; Elles, C. G.; Scopigno, T. Spectrally tailored narrowband pulses for femtosecond stimulated Raman spectroscopy in the range 330-750 nm. Opt. Express 2013, 21, 6866-6872.

(58) Hoffman, D. P.; Valley, D.; Ellis, S. R.; Creelman, M.; Mathies, R. A. Optimally shaped narrowband picosecond pulses for femtosecond stimulated Raman spectroscopy. Opt. Express 2013, 21, 21685.

(59) Batignani, G.; Fumero, G.; Pontecorvo, E.; Ferrante, C.; Mukamel, S.; Scopigno, T. Genuine dynamics vs cross phase modulation artefacts in Femtosecond Stimulated Raman Spectroscopy. ACS Photonics 2019, 6, 492-500.

(60) Ferrante, C.; Batignani, G.; Pontecorvo, E.; Montemiglio, L. C.; Vos, M. H.; Scopigno, T. Ultrafast dynamics and vibrational relaxation in six-coordinate heme proteins revealed by Femtosecond Stimulated Raman Spectroscopy. J. Am. Chem. Soc. 2020, 142, 2285-2292.

(61) Batignani, G.; Fumero, G.; Mukamel, S.; Scopigno, T. Energy flow between spectral components in $2 \mathrm{D}$ broadband stimulated Raman spectroscopy. Phys. Chem. Chem. Phys. 2015, 17, 10454-10461.

(62) Agarwalla, B. K.; Ando, H.; Dorfman, K. E.; Mukamel, S. Stochastic Liouville equations for femtosecond stimulated Raman spectroscopy. J. Chem. Phys. 2015, 142, 024115.

(63) Dorfman, K. E.; Fingerhut, B. P.; Mukamel, S. Time-resolved broadband Raman spectroscopies: A unified six-wave-mixing representation. J. Chem. Phys. 2013, 139, 124113.

(64) Batignani, G.; Ferrante, C.; Fumero, G.; Scopigno, T. Broadband impulsive stimulated Raman scattering based on a chirped detection. J. Phys. Chem. Lett. 2019, 10, 7789-7796.

(65) Becke, A. D. Density-functional exchange-energy approximation with correct asymptotic behavior. Phys. Rev. A: At., Mol., Opt. Phys. 1988, 38, 3098-3100.

(66) Lee, C.; Yang, W.; Parr, R. G. Development of the Colle-Salvetti correlation-energy formula into a functional of the electron density. Phys. Rev. B: Condens. Matter Mater. Phys. 1988, 37, 785-789. 